\title{
Asymptotically Matched Layer (AML) for transient wave propagation in a moving frame of reference
}

\author{
Madsen, Stine Skov; Krenk, Steen
}

Published in:

Computers and Geotechnics

Link to article, DOI:

10.1016/j.compgeo.2016.09.005

Publication date:

2017

Document Version

Peer reviewed version

Link back to DTU Orbit

Citation (APA):

Madsen, S. S., \& Krenk, S. (2017). Asymptotically Matched Layer (AML) for transient wave propagation in a moving frame of reference. Computers and Geotechnics, 82, 124-133.

https://doi.org/10.1016/j.compgeo.2016.09.005

\section{General rights}

Copyright and moral rights for the publications made accessible in the public portal are retained by the authors and/or other copyright owners and it is a condition of accessing publications that users recognise and abide by the legal requirements associated with these rights.

- Users may download and print one copy of any publication from the public portal for the purpose of private study or research.

- You may not further distribute the material or use it for any profit-making activity or commercial gain

- You may freely distribute the URL identifying the publication in the public portal

If you believe that this document breaches copyright please contact us providing details, and we will remove access to the work immediately and investigate your claim 


\title{
Asymptotically Matched Layer (AML) for transient wave propagation in a moving frame of reference
}

\author{
Stine Skov Madsen ${ }^{1,2}$, Steen Krenk ${ }^{2}$ \\ ${ }^{1}$ Geotechnics and Geology, Department of Civil Engineering, Technical University of Denmark \\ ${ }^{2}$ Solid Mechanics, Department of Mechanical Engineering, Technical University of Denmark
}

\begin{abstract}
The paper presents an Asymptotically Matched Layer (AML) formulation in a moving frame of reference for transient dynamic response of a multi-layer 2D half-space. A displacement based finite element formulation of the convected domain problem is presented together with the AML formulation in which the original convolution integrals are represented via two auxiliary displacement-like state-space variables. A parametric study of the AML parameters is conducted for optimizing the absorbing properties. The performance is demonstrated on a single- and a two-layered halfspace for various velocities of an impulse Ricker load. Excellent absorbing properties are demonstrated in both half spaces.

Keywords: Convected coordinates, Finite element method, Absorbing boundary, PML, Moving load, Transient wave propagation
\end{abstract}

\section{Introduction}

Dynamic analysis of moving loads is of great interest in the fields of road and railway traffic. Determination of the dynamic response of road and airport pavements to moving dynamic loads is very important in pavement design in particular to predict road damage. With the increasing interest of high-speed train lines it 
has as well become important to understand the dynamic behavior of a multi-layer half-space subjected to moving loads.

Modeling of elastic wave propagation in a half space requires accurate boundary conditions to allow only outgoing waves. In many cases a numerical solution is developed by applying the finite element method over the computational domain. In the case of a half space, the unbounded region must be truncated to a computational domain of interest, including suitable boundary conditions that are transparent to incident waves, thereby representing the unbounded region.

A technique that has demonstrated very high efficiency as absorbing boundary is the Perfectly Matched Layer (PML). The PML was first suggested by Berenger [1] for the absorption of electromagnetic waves. Shortly after new interpretations to this method was suggested by Chew [2] in terms of introducing the stretch coordinate. Chew and Liu [3] proved that a perfectly matched layer is also applicable for elastic waves using the method of complex coordinate stretching. Later the PML technique was formulated for the elastic wave equation using several modifications by e.g. [4, $5,6]$. However, in these approaches the solution for the displacements is dependent on computation of the strains in each time step. This problem was circumvented in a combined stress-displacement formulation presented by Kucukcoban and Kallivokas [7]. A simpler procedure, depending only on the displacements, using an artificially anisotropic material description of the PML layer, was recently proposed by Matzen [8]. This method proved to be highly efficient, when solving transient vibration problems in a stationary frame of reference.

Potential stability problems of the PML layer formulation for stationary anisotropic problems and wave guides have been discussed in $[9,10,11]$. The problems are related to the basic assumption of the PML layer, that waves propagate into the 
layer via impedance matching and are damped while they propagate. An extensive survey of the various PML formulations has been given in [7].

In some studies the dynamic response of a layered half space subjected to a moving load is obtained by manually moving the load from element to element. This approach is used by e.g. Yoo and Al-Qadi [12] in their study of transient dynamic loading of a pavement using a 3D finite element model and Wang et al. [13] analyzing the pavement response of an instrumented runway under moving aircraft tire loading.

Lane et al. [14] presented a mesh movement algorithm where the elements are moved with the same velocity as the load in their study of dynamic vehicle interaction and wave propagation in a 3D finite element model. This requires a change of the grid in each time step. On the other hand, Dieterman and Metrikine [15] studied the analytical solution of the steady-state displacement of an EulerBernoulli beam resting on an elastic half space due to a uniformly constant moving load by introducing a coordinate transformation into a moving frame of reference. Krenk et al. [16] presented 2D formulation in a convected coordinate system moving with the load and using a transmitting boundary condition in the form of a springdashpot model for absorption of waves, modified to account for the translation velocity and the difference between compression and shear waves, see also [17]. The advantage of a transformation into moving coordinates is that a high mesh resolution can be concentrated around the load without any need of remeshing. This is especially important in 3D finite element modeling where computation time increases rapidly with increasing amount of elements.

In this paper, a formulation of an Asymptotically Matched Layer (AML) is developed in a moving frame of reference based on the PML formulation in [8]. The 
formulation essentially is a transformation of the PML scheme into moving coordinates, but with the damping terms from the constitutive part of the equation represented in the moving coordinate system in order to facilitate the evaluation of the integral representation of the damping terms. In [18] an improved PML formulation was presented for the acoustic problem, based on a coordinate transformation in the PML layer incorporating the direction dependence of the wave slowness diagram, but this techniques can not account for the different shear and compression wave speeds. The present formulation is given in $2 \mathrm{D}$ but it can be expanded to 3D as well. The procedure uses displacement based finite elements and represents the AML memory effect via two displacement-like auxiliary state-space variables. A parametric study of the AML parameters is conducted for optimizing the performance. Numerical examples show the response from a Ricker impulse load of various velocity obtained in two points placed behind and in front of the load, respectively, with equal distance to the load. The absorption abilities of the AML is evaluated in a single as well as a two layer half space. The effect of stiffness ratio between the top layer and the underlying layer in a 2-layer system is studied as well.

\section{Perfectly Matched Layer (PML)}

The present section introduces the method of a perfectly matched layer for absorbing out-going elastic waves. The method, introduced in [8], makes use of a formal coordinate transformation by which a regular wave in the transformed spatial coordinates is recast into an equivalent problem in the original coordinates in such a way that the coordinate transformation appears as coefficients in the governing equations that are subsequently solved by finite elements. The characteristic feature of the method is that the transformation is only introduced in a layer sur- 
rounding the computational domain, in which the transformation degenerates to an identity. Thus, the special features of the absorbing boundary condition only appear in the surrounding layer, which is included in the finite element model. The boundary layer is characterized by its thickness and parameters describing its dissipation properties.

\subsection{Two-dimensional wave propagation}

The goal now is to set up a set of equations for a stretched 2D elasticity problem that incorporates damping if the coordinates are stretched, and specializes to the classic undamped elasticity equations if the original coordinates are retained without stretching. The equations of two-dimensional isotropic linear elasticity consist of the constitutive equations and the equations of motion. The constitutive equations relating the stresses $\boldsymbol{\sigma}$ and the derivatives of the displacements $\mathbf{u}$ are

$$
\boldsymbol{\sigma}=\lambda\left(\boldsymbol{\nabla}^{T} \mathbf{u}\right) \mathbf{I}+\mu\left[\left(\boldsymbol{\nabla} \mathbf{u}^{T}\right)+\left(\boldsymbol{\nabla} \mathbf{u}^{T}\right)^{T}\right]
$$

where the gradient operator is defined by

$$
\boldsymbol{\nabla}=\left[\frac{\partial}{\partial x_{1}}, \frac{\partial}{\partial x_{2}}\right]^{T}
$$

and $\lambda$ and $\mu$ are the Lamé parameters. When considering harmonic time variation represented via the factor $\exp (i \omega t)$, the equation of motion takes the form

$$
\left(\nabla^{T} \boldsymbol{\sigma}\right)^{T}=-\omega^{2} \rho \mathbf{u}
$$

where $\rho$ is the mass density.

The original elasticity problem is now reformulated by using the notion of stretched coordinates. The idea is to introduce a set of stretched coordinates $\tilde{x}_{j}=\tilde{x}_{j}\left(x_{j}\right)$, 
defined in terms of the original coordinates $x_{j}$ by the relation

$$
\frac{\partial \tilde{x}_{j}}{\partial x_{j}}=s_{j}, \quad j=1,2
$$

for the derivatives. It is noted that by this assumption each coordinate is stretched independently. In the present paper the stretching functions $s_{1}$ and $s_{2}$ are chosen in the form

$$
s_{j}\left(x_{j}\right)=1+\frac{\beta_{j}\left(x_{j}\right)}{i \omega}, \quad j=1,2 .
$$

The stretching functions deviate from unity by an additive term consisting of an attenuation function $\beta_{j}\left(x_{j}\right)$ depending on the coordinate $x_{j}$ and assumed increasing through the bounding layer. The attenuation function is divided by the imaginary frequency factor $i \omega$. When converting the frequency representation to the time domain, the frequency factor $(i \omega)^{-1}$ corresponds to time integration in the same way the factor $i \omega$ corresponds to time differentiation. The role of the attenuation functions is to introduce an imaginary part that increases gradually from the interface between the elastic domain and the surrounding boundary layer. In the present paper this is accomplished by selecting the attenuation functions in the form, [19, 20],

$$
\beta_{j}\left(x_{j}\right)=\beta_{\max }\left(\frac{x_{j}^{p}}{d}\right)^{2}
$$

where the superscript $p$ denotes the corresponding coordinate with origin at the interface between the elastic domain and the boundary layer. The boundary layer surrounds the elastic region as illustrated in Figure 1 showing three side regions and two corner regions. In the side regions only the coordinate orthogonal to the interface is transformed, while both coordinates are transformed in the corner regions.

The idea now is to formulate a formal elasticity problem by using derivatives in terms of the stretched coordinates, and by introducing a suitable formal stress 


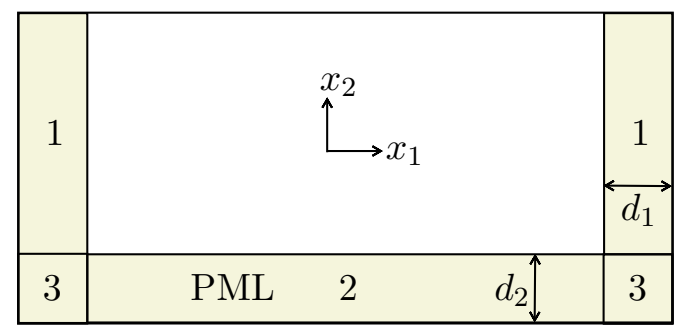

Figure 1: Region 1: $s_{1}=1+\beta_{1} / i \omega$ and $s_{2}=1$, region $2: s_{1}=1$ and $s_{2}=1+\beta_{2} / i \omega$, region 3 : $s_{1}=1+\beta_{1} / i \omega$ and $s_{2}=1+\beta_{2} / i \omega$. In the computational domain $s_{1}=s_{2}=1$.

definition. Once the equations are formulated, the stretching parameters are absorbed into the constitutive parameters and the mass density corresponding to time differentiation and integration operators on the physical parameters $\lambda, \mu$ and $\rho$. The first step is to introduce the transformed gradient operator

$$
\tilde{\nabla}=\left[\frac{\partial}{\partial \tilde{x}_{1}}, \frac{\partial}{\partial \tilde{x}_{2}}\right]^{T}=\left[\frac{1}{s_{1}} \frac{\partial}{\partial x_{1}}, \frac{1}{s_{2}} \frac{\partial}{\partial x_{2}}\right]^{T}
$$

in terms of the stretched coordinates. In the boundary layer the use of this gradient operator would define a formal stress

$$
\boldsymbol{\sigma}=\lambda\left(\tilde{\nabla}^{T} \mathbf{u}\right) \mathbf{I}+\mu\left[\left(\tilde{\nabla} \mathbf{u}^{T}\right)+\left(\tilde{\nabla} \mathbf{u}^{T}\right)^{T}\right] .
$$

It follows from the format of this formal stress definition that the stress component matrix is symmetric, $\sigma_{12}=\sigma_{21}$. However, the formal strain matrix, given by the square brackets in (8), is now no longer symmetric, and the off-diagonal elements are defined in terms of the classic shear strain as well as the rotation. Thus, there are essentially four deformation components, but only three components in the formal stress matrix $\boldsymbol{\sigma}$. A resolution to the problem is suggested by the formal equation of motion,

$$
\left(\tilde{\nabla}^{T} \boldsymbol{\sigma}\right)^{T}=-\omega^{2} \rho \mathbf{u}
$$


The transformation of the gradient operator introduces factors $s_{j}^{-1}$ on the derivatives corresponding to the first index of $\sigma_{j k}$. This suggests the use of a formal stress with components defined by, [3],

$$
\tilde{\boldsymbol{\sigma}}=s_{1} s_{2}\left[\begin{array}{ll}
1 / s_{1} & \\
& 1 / s_{2}
\end{array}\right] \boldsymbol{\sigma} .
$$

The factor $s_{1} s_{2}$ in this relation is suggested by the consideration that the final form of the formal stress-strain relation should not contain powers of $s_{j}$ less than of degree -1 in order to enable a direct interpretation of the frequency problem in the time domain as discussed later.

When introducing the definition (10), the formal stress $\tilde{\boldsymbol{\sigma}}$ is related to a set of formal strains including the rotation component by a relation of the form

$$
\tilde{\boldsymbol{\sigma}}=\tilde{\mathbf{C}} \varepsilon
$$

In this relation it is convenient to introduce the formal stress in the array format

$$
\overline{\boldsymbol{\sigma}}=\left[\begin{array}{c}
\tilde{\sigma}_{11} \\
\tilde{\sigma}_{22} \\
\frac{1}{2}\left(\tilde{\sigma}_{21}+\tilde{\sigma}_{12}\right) \\
\frac{1}{2}\left(\tilde{\sigma}_{21}-\tilde{\sigma}_{12}\right)
\end{array}\right]
$$

and the formal strains in the corresponding array format

$$
\boldsymbol{\varepsilon}=\boldsymbol{\partial u}=\left[\begin{array}{cc}
\partial_{x_{1}} & 0 \\
0 & \partial_{x_{2}} \\
\partial_{x_{2}} & \partial_{x_{1}} \\
\partial_{x_{2}} & -\partial_{x_{1}}
\end{array}\right]\left[\begin{array}{l}
u_{1} \\
u_{2}
\end{array}\right]
$$


Straightforward substitution of (10) into the constitutive equations (8) then gives the constitutive matrix $\tilde{\mathbf{C}}$ in the form

$$
\tilde{\mathbf{C}}=\mathbf{C}_{0}+\frac{s_{1}}{s_{2}} \mathbf{C}_{1}+\frac{s_{2}}{s_{1}} \mathbf{C}_{2}
$$

The non-stretched part $\mathbf{C}_{0}$ and the two stretched parts $\mathbf{C}_{1}$ and $\mathbf{C}_{2}$ are given by

$$
\begin{gathered}
\mathbf{C}_{0}=\left[\begin{array}{cccc}
0 & \lambda & 0 & 0 \\
\lambda & 0 & 0 & 0 \\
0 & 0 & \mu / 2 & 0 \\
0 & 0 & 0 & -\mu / 2
\end{array}\right], \quad \mathbf{C}_{1}=\left[\begin{array}{cccc}
0 & 0 & 0 & 0 \\
0 & \lambda+2 \mu & 0 & 0 \\
0 & 0 & \mu / 4 & \mu / 4 \\
0 & 0 & \mu / 4 & \mu / 4
\end{array}\right], \\
\mathbf{C}_{2}=\left[\begin{array}{cccc}
\lambda+2 \mu & 0 & 0 & 0 \\
0 & 0 & 0 & 0 \\
0 & 0 & \mu / 4 & -\mu / 4 \\
0 & 0 & -\mu / 4 & \mu / 4
\end{array}\right]
\end{gathered}
$$

In the special case $s_{1}=s_{2}=1$, used in the computational domain, the matrix takes the form

$$
\mathbf{C}=\mathbf{C}_{0}+\mathbf{C}_{1}+\mathbf{C}_{2}
$$

corresponding to plane strain with symmetric stress components.

The equation of motion is obtained from (10), when disregarding the spatial derivatives of the factors $s_{1}$ and $s_{2}$. Hereby the equations of motion in terms of the formal stress $\overline{\boldsymbol{\sigma}}$ in the array format (12) take the form

$$
\boldsymbol{\partial}^{T} \overline{\boldsymbol{\sigma}}=-\rho s_{1} s_{2} \omega^{2} \mathbf{u}
$$

where $\boldsymbol{\partial}$ is the spatial differential operator introduced in the strain definition (13). 


\subsection{Time domain equations}

The frequency-dependent system of equations consisting of the constitutive equation (11) is transformed into time domain using the inverse Fourier transform. The constitutive equation takes the form

$$
\overline{\boldsymbol{\sigma}}=\mathcal{C} * \varepsilon
$$

where the symbol $*$ implies convolution with the time-dependent constitutive matrix defined by

$$
\mathcal{C}=\mathbf{C}+\mathcal{F}_{1}(t) \mathbf{C}_{1}+\mathcal{F}_{2}(t) \mathbf{C}_{2}
$$

It is noted that the matrix $\mathbf{C}$ corresponds to the standard time-independent form defined in (16). Thus, the functions $\mathcal{F}_{1}(t)$ and $\mathcal{F}_{2}(t)$ are the inverse Fourier transforms of $s_{1} / s_{2}-1$ and $s_{2} / s_{1}-1$, respectively,

$$
\begin{aligned}
& \mathcal{F}_{1}(t)=\left(\beta_{1}-\beta_{2}\right) e^{-\beta_{2} t}, \quad t \geq 0 \\
& \mathcal{F}_{2}(t)=\left(\beta_{2}-\beta_{1}\right) e^{-\beta_{1} t}, \quad t \geq 0
\end{aligned}
$$

The implementation of this formulation makes use of a time-step form in which the convolution integrals involving $\mathcal{F}_{1}(t)$ and $\mathcal{F}_{2}(t)$ are replaced by increments, thereby limiting the computations to the current time increment.

In the time domain the equation of motion (17) takes the form

$$
\partial^{T} \overline{\boldsymbol{\sigma}}=\mathcal{D}_{0}(t) \rho \mathbf{u}
$$

The operator $\mathcal{D}_{0}(t)$ is the inverse Fourier transform of $-\omega^{2} s_{1} s_{2}$ given by

$$
\mathcal{D}_{0}(t)=\frac{d^{2}}{d t^{2}}+\left(\beta_{1}+\beta_{2}\right) \frac{d}{d t}+\beta_{1} \beta_{2}
$$

The first term represents the inertia term, while the second term represents a velocity proportional viscous damping and the last term a mass-proportional stiffness, all acting on the absolute motion. 


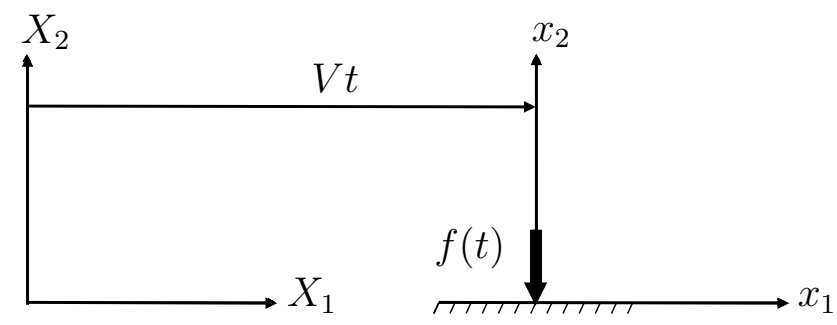

Figure 2: Moving surface load in fixed $X_{i}$-coordinate system

\section{Convected Mesh Model}

Following Krenk et al. [16] a convected coordinate system moving with the load is introduced via the relation

$$
x=X-V t
$$

where $X$ is the coordinate of the moving load in the fixed reference coordinate system, while $x$ is the coordinate in the coordinate system following the load that is moving with velocity $V$. The relation (23) implies the following differentiation relations

$$
\left.\frac{\partial}{\partial X}\right|_{t}=\left.\frac{\partial}{\partial x}\right|_{t},\left.\quad \frac{\partial}{\partial t}\right|_{X}=\left.\frac{\partial}{\partial t}\right|_{x}-V \frac{\partial}{\partial x}
$$

Note that for convenience $x$ is used instead of $x_{1}$ in the derivative with respect to $x_{1}$.

Substitution of these operators into the equation of motion (21) leads to the following modified form of the equilibrium equation in the moving coordinate system

$$
\partial^{T} \overline{\boldsymbol{\sigma}}=\tilde{\mathcal{D}}_{0}(t) \rho \mathbf{u}
$$

with the convected time differentiation operator

$$
\overline{\mathcal{D}}_{0}(t)=\left(\frac{\partial}{\partial t}-V \frac{\partial}{\partial x}\right)^{2}+\left(\beta_{1}+\beta_{2}\right)\left(\frac{d}{d t}-V \frac{d}{d x}\right)+\beta_{1} \beta_{2}
$$


When using this operator in the dynamic equation (25) the following form of the equation is obtained

$$
\begin{aligned}
\boldsymbol{\partial}^{T} \overline{\boldsymbol{\sigma}}= & \rho\left(\frac{\partial^{2} \mathbf{u}}{\partial t^{2}}+\left(\beta_{1}+\beta_{2}\right) \frac{\partial \mathbf{u}}{\partial t}+\beta_{1} \beta_{2} \mathbf{u}\right) \\
& +V^{2} \frac{\partial^{2}(\rho \mathbf{u})}{\partial x^{2}}-\left(\beta_{1}+\beta_{2}\right) V \frac{\partial(\rho \mathbf{u})}{\partial x}-2 V \frac{\partial^{2}(\rho \mathbf{u})}{\partial x \partial t}
\end{aligned}
$$

The first three terms on the right correspond to the representation in a fixed coordinate system, while the three last represent the effect of translation.

The convolution integrals in the constitutive relation (18) are related to the artificial properties of the boundary layer, and when assuming these properties convected with the load the form of the constitutive equation remains unchanged by the translation. Hereby the transformation from fixed to moving coordinates only modifies the dynamic equation, permitting a fairly straight forward implementation of the AML formulation in the translating formulation.

\section{Finite element implementation}

The principle of virtual work is used to obtain the weak formulation of the equation of motion (25), yielding

$$
\int_{V} \tilde{\mathbf{u}}^{T}\left(\boldsymbol{\partial}^{T} \overline{\boldsymbol{\sigma}}\right) d V-\int_{V} \tilde{\mathbf{u}}^{T} \tilde{\mathcal{D}}_{0}(t) \rho \mathbf{u} d V=0
$$

The spatial variation of the actual and the virtual displacement fields are represented by shape functions as

$$
\begin{aligned}
& \mathbf{u}(\mathbf{x}, t)=\mathbf{N}(\mathbf{x}) \mathbf{d}(t) \\
& \tilde{\mathbf{u}}(\mathbf{x}, t)=\tilde{\mathbf{N}}(\mathbf{x}) \tilde{\mathbf{d}}(t)
\end{aligned}
$$


with the shape function matrix $\mathbf{N}(\mathbf{x})$ in the form

$$
\mathbf{N}=\left[\begin{array}{ccccccc}
N_{1} & 0 & N_{2} & 0 & \cdots & N_{n} & 0 \\
0 & N_{1} & 0 & N_{2} & \cdots & 0 & N_{n}
\end{array}\right]
$$

and $\tilde{\mathbf{N}}$ on a similar form.

The shape functions are inserted in (28) which is reformulated using integration by parts in order to obtain a symmetric formulation

$$
\begin{aligned}
& \int_{V}\left\{\tilde{\boldsymbol{\varepsilon}}^{T} \overline{\boldsymbol{\sigma}}+\rho \tilde{\mathbf{u}}^{T} \ddot{\mathbf{u}}-\rho V\left(\tilde{\mathbf{u}}^{T} \frac{\partial \dot{\mathbf{u}}}{\partial x}-\dot{\mathbf{u}}^{T} \frac{\partial \tilde{\mathbf{u}}}{\partial x}\right)+\rho V^{2} \frac{\partial \tilde{\mathbf{u}}^{T}}{\partial x} \frac{\partial \mathbf{u}}{\partial x}+\rho\left(\beta_{1}+\beta_{2}\right) \tilde{\mathbf{u}}^{T} \dot{\mathbf{u}}\right. \\
& \left.+\frac{1}{2} \rho V\left(\beta_{1}+\beta_{2}\right)\left(\mathbf{u}^{T} \frac{\partial \tilde{\mathbf{u}}}{\partial x}-\tilde{\mathbf{u}}^{T} \frac{\partial \mathbf{u}}{\partial x}\right)+\rho \beta_{1} \beta_{2} \tilde{\mathbf{u}}^{T} \mathbf{u}\right\} d V \\
& =\int_{S}\left\{\tilde{\mathbf{u}}^{T} \boldsymbol{\sigma} \boldsymbol{n}+\rho V \tilde{\mathbf{u}}^{T}\left(\dot{\mathbf{u}}-V \frac{\partial \mathbf{u}}{\partial x}\right) \mathbf{n}_{x}+\frac{1}{2} \rho V\left(\beta_{1}+\beta_{2}\right) \tilde{\mathbf{u}}^{T} \mathbf{u} \mathbf{n}_{x}\right\} d S
\end{aligned}
$$

The load is traveling in the $x_{1}$-direction, and when assuming full attenuation within the AML-layer the surface is the only free boundary. Thus, the two last terms in the surface integral vanish.

Separating the convolution terms in the operators $\mathcal{F}_{1}(t)$ and $\mathcal{F}_{2}(t)$ in the constitutive matrix $\mathcal{C}$, the following set of ordinary differential equations is obtained

$$
\mathbf{M} \ddot{\mathbf{u}}+\mathbf{Z} \dot{\mathbf{u}}+\mathbf{K u}+\mathbf{g}=\mathbf{f}
$$

where $\mathbf{u}$ is the global displacement vector and $\mathbf{f}$ is the global force vector, assumed to represent surface loads, whereby

$$
\mathbf{f}=\int_{S} \tilde{\mathbf{N}}^{T} \boldsymbol{\sigma} \mathbf{n} d S
$$


The element mass, damping and stiffness matrices are given by

$$
\begin{aligned}
\mathbf{M}= & \int_{V} \rho \tilde{\mathbf{N}}^{T} \mathbf{N} d V \\
\mathbf{Z}= & \int_{V}-\rho V\left(\tilde{\mathbf{N}}_{x}^{T} \mathbf{N}-\hat{\mathbf{N}}^{T} \mathbf{N}_{x}\right)+\rho\left(\beta_{1}+\beta_{2}\right) \tilde{\mathbf{N}}^{T} \mathbf{N} d V \\
\mathbf{K}= & \int_{V}\left\{\tilde{\mathbf{B}}^{T} \mathbf{C B}-\rho V^{2} \tilde{\mathbf{N}}_{x}^{T} \mathbf{N}_{x}\right. \\
& \left.+\frac{1}{2} \rho V\left(\beta_{1}+\beta_{2}\right)\left(\tilde{\mathbf{N}}_{x}^{T} \mathbf{N}-\tilde{\mathbf{N}}^{T} \mathbf{N}_{x}\right)+\rho \beta_{1} \beta_{2} \tilde{\mathbf{N}}^{T} \mathbf{N}\right\} d V
\end{aligned}
$$

where $\mathbf{B}$ denotes the strain-displacement matrix and $\mathbf{N}$ the shape functions with $x$-derivative $\mathbf{N}_{x}=\partial \mathbf{N} / \partial x$.

Following [8] the convolution integrals from the constitutive relation, representing artificial damping in the AML, are now associated with the nodal displacements, whereby the corresponding vector $\mathrm{g}$ takes the form

$$
\mathbf{g}^{e}=\mathbf{K}_{1}^{e} \mathcal{F}_{1} * \mathbf{u}(t)+\mathbf{K}_{2}^{e} \mathcal{F}_{2} * \mathbf{u}(t)
$$

with element matrices $\mathbf{K}_{1}$ and $\mathbf{K}_{2}$ given by

$$
\mathbf{K}_{p}^{e}=-\int_{\Omega} \mathbf{B}^{T} \mathbf{C}_{p} \mathbf{B} d V, \quad p=1,2
$$

By this, approximate, procedure the convolution terms $\mathcal{F}_{p} * \mathbf{u}(t)$ are defined using the value of the AML parameters at the nodes. The general appearance of the convolution term is

$$
\mathcal{F}_{p} * \mathbf{u}(t)=\int_{0}^{t} \mathcal{F}_{p}(\tau) \mathbf{u}(t-\tau) d \tau=\int_{0}^{t}\left(\beta_{\bar{p}}-\beta_{p}\right) e^{-\beta_{p} \tau} \mathbf{u}(t-\tau) d \tau
$$

with index $\bar{p}$ being the complement of $p$. In the integral the arguments $\tau$ and $t-\tau$ can be interchanged, and differentiation with respect to $t$ then leads to the first-order 
'filter type' equation

$$
\frac{d}{d t}\left(\mathcal{F}_{p} * \mathbf{u}(t)\right)+\beta_{p}\left(\mathcal{F}_{p} * \mathbf{u}(t)\right)=\left(\beta_{\bar{p}}-\beta_{p}\right) \mathbf{u}(t)
$$

When using this form, the functions $\mathbf{v}_{p}(t)=\mathcal{F}_{p} * \mathbf{u}(t)$, with dimension of displacement, can be considered as state-space variables and can be updated explicitly via a finite difference approximation as indicated in the description of the numerical algorithm in section 4.2. This constitutes a simple alternative to the procedure in [7] using a combined displacement stress-field representation. It may be argued that the time derivative in the filter equation (39) should be modified to account for the moving frame. However, the fading memory is fictitious and need not be implemented in a stationary frame, and furthermore the representation of the spatial variation of the AML parameters has already been replaced by their nodal histories by $(36)$.

\subsection{Correction for moving frame}

The translation of the coordinate system leads to terms proportional to $V$ and $V^{2}$ in (27). These terms make the equations loose the original self-adjointness. In principle this can be compensated for by a modification of the shape functions. However, in the present problem it is simpler to use a technique developed by Krenk et al. [16] in which the terms $2 \rho V \dot{\mathbf{u}}_{x}$ and $\rho V\left(\beta_{1}+\beta_{2}\right) \mathbf{u}_{x}$ are modified to account for the convection effect. A Taylor series expansion demonstrates that a straightforward Galerkin representation of these terms implies an error illustrated by the two-term Taylor expansion

$$
\dot{\mathbf{u}}_{x} \simeq \frac{-\Delta \dot{\mathbf{u}}}{h}+\frac{1}{2} h \dot{\mathbf{u}}_{x x}
$$

where $h$ denotes the length of the increment $\Delta x$ in the opposite direction of the load velocity. The first term on the right hand side of (40) is already properly represented 
by linear interpolation, hence the second term should be inserted in (27) for an improved formulation. The same procedure is used for the term $\rho V\left(\beta_{1}+\beta_{2}\right) \mathbf{u}_{x}$ and insertion of the terms of improvement in Eq. (27) yields

$$
\begin{aligned}
\partial^{T} \overline{\boldsymbol{\sigma}}-\rho & \left(\frac{\partial^{2} \mathbf{u}}{\partial t^{2}}+V^{2} \frac{\partial^{2}\left(\mathbf{u}-(h / V) \dot{\mathbf{u}}-\frac{1}{2}(h / V)\left(\beta_{1}+\beta_{2}\right) \mathbf{u}\right)}{\partial x^{2}}\right. \\
& \left.-2 V \frac{\partial \dot{\mathbf{u}}}{\partial x}+\left(\beta_{1}+\beta_{2}\right) \frac{\partial \mathbf{u}}{\partial t}-\left(\beta_{1}+\beta_{2}\right) V \frac{\partial \mathbf{u}}{\partial x}+\beta_{1} \beta_{2} \mathbf{u}\right)
\end{aligned}
$$

The introduction of the correction terms yields an extra contribution to the volume terms of the damping and stiffness matrix. The additional volume damping and stiffness matrices are

$$
\begin{aligned}
\mathbf{Z}_{V} & =h \int_{V} \rho V \tilde{\mathbf{N}}_{x}^{T} \mathbf{N}_{x} d V \\
\mathbf{K}_{V} & =h \int_{V} \frac{1}{2} \rho V\left(\beta_{1}+\beta_{2}\right) \tilde{\mathbf{N}}_{x}^{T} \mathbf{N}_{x} d V
\end{aligned}
$$

The effect of the improved formulation is an additional convection term proportional to the second order derivative in space added to the full system in terms of damping and to the boundary layer in terms of stiffness. A suitable value for the convection correction parameter $h$ was found by Krenk et al. [16] to be around $0.3-0.4$ times the length of the elements in the $x$-direction.

\subsection{Time integration}

The FE discretized system of equations are integrated in time using the NewmarkBeta time integration method [21]. The result is the following time marching of the 


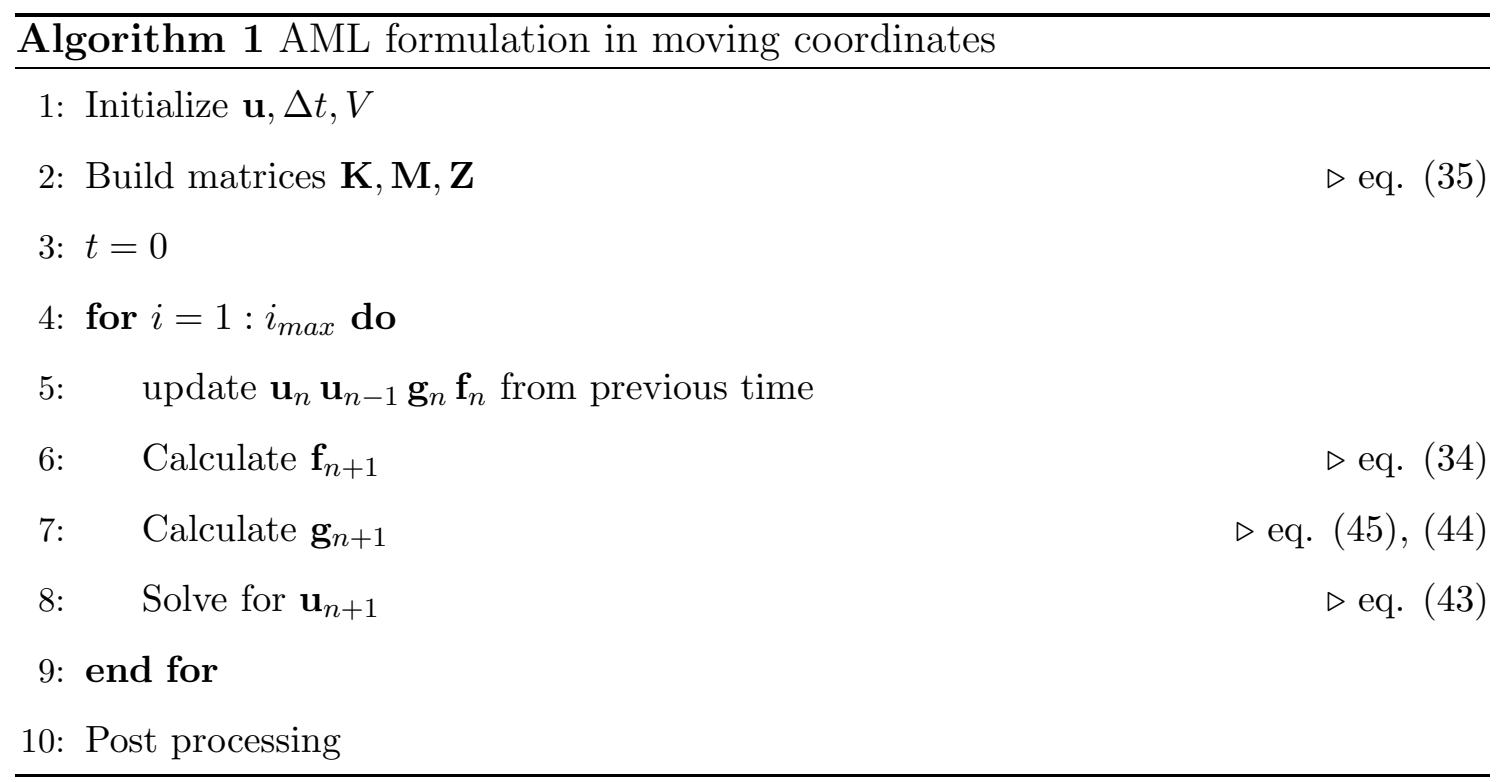

elastic displacement field

$$
\begin{aligned}
& \left(\frac{1}{\Delta t^{2}} \mathbf{M}+\frac{1}{2 \Delta t} \mathbf{Z}+\beta \mathbf{K}\right) \mathbf{u}^{n+1} \\
& =\left(\frac{2}{\Delta t^{2}} \mathbf{M}-(1-2 \beta) \mathbf{K}\right) \mathbf{u}^{n}-\left(\frac{1}{\Delta t^{2}} \mathbf{M}-\frac{1}{2 \Delta t} \mathbf{Z}+\beta \mathbf{K}\right) \mathbf{u}^{n-1} \\
& \quad-\beta \mathbf{g}^{n+1}-(1-2 \beta) \mathbf{g}^{n}-\beta \mathbf{g}^{n-1}+\beta \mathbf{f}^{n+1}+(1-2 \beta) \mathbf{f}^{n}+\beta \mathbf{f}^{n-1}
\end{aligned}
$$

The parameter, $\beta$, controls the interpolation between explicit and implicit time integration schemes. It has been shown that the integration scheme becomes unconditionally stable when $\beta \geq 1 / 4$. As opposed to explicit methods in which the time step $\Delta t$ is bounded by the CFL condition, the implicit method has no limiting time step. However, the time step should be chosen such that a minimum resolution of wave propagation is present. Zhai and Song [22] suggest a minimum time step of $\Delta t=1 /\left(8 f_{\max }\right)$ where $f_{\max }$ is the largest frequency present. 
The value of $\mathbf{g}^{n+1}$ follows from (36), when expressed in the form

$$
\mathbf{g}^{n+1}=\mathbf{K}_{1} \mathbf{v}_{1}^{n+1}+\mathbf{K}_{2} \mathbf{v}_{2}^{n+1}
$$

with the auxiliary state-space vectors $\mathbf{v}_{p}^{n+1}$ determined from a finite difference form of the filter equations (39). A particularly simple form is a central difference around $t_{n}$ and weight parameter $\beta$ in the form

$$
\frac{1}{2 \Delta t}\left(\mathbf{v}_{p}^{n+1}-\mathbf{v}_{p}^{n-1}\right)+\beta_{p}\left(\beta \mathbf{v}_{p}^{n+1}+(1-2 \beta) \mathbf{v}_{p}^{n}+\beta \mathbf{v}_{p}^{n-1}\right)=\left(\beta_{\bar{p}}-\beta_{p}\right) \mathbf{u}^{n}
$$

This equation determines $\mathbf{v}_{1}^{n+1}$ and $\mathbf{v}_{2}^{n+1}$ leading to $\mathbf{g}^{n+1}$ by (44). For the discretization of the AML and the computational domain quadrilateral bilinear elements are employed. The procedure is presented in Algorithm 1.

\section{Numerical Examples}

In this section, numerical examples are conducted to demonstrate the absorbing properties of the AML formulated in a moving frame of reference. In that sense the Ricker pulse is used as an example of a moving source. In section 5.1, the AML boundary condition is verified against a large domain and the results are compared to a solution obtained by substituting the AML layer with fixed boundaries. The wave propagation is visualized with snapshots at four instances of time with AML boundaries and fixed boundaries, respectively. The simulation in a moving frame of reference is verified against a manually moved load in a static frame of reference. In section 5.2 a parametric study on the AML parameters is conducted. In section 5.3 and 5.4 the AML is used to study the response of a Ricker pulse traveling across the surface of a single- and a two-layer half space, respectively, with different velocities. Section 5.3 treats a single layer half space and demonstrates the change in response 
with velocity in front of and behind the load. Section 5.4 investigates the effect of putting a stiff layer on top of the soft subgrade for varying impedances.

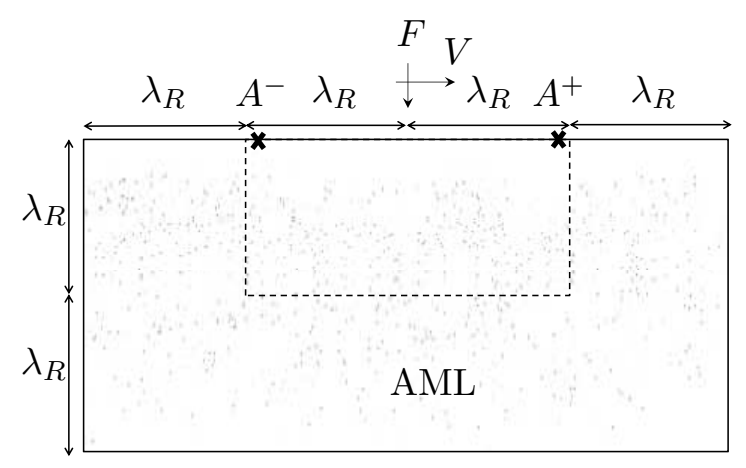

Figure 3: Domain of interest truncated by AML.

The numerical examples are based on the half space sketched in Figure 3. The dashed line indicates the interface between the computational domain and the AML. $F$ is the load acting at the center of the surface and $V$ indicates the velocity and traveling direction of the load. In all numerical examples, the computational domain has a width of $2 \lambda_{R}$ and a depth of $\lambda_{R}$, where $\lambda_{R}$ is the Rayleigh wave length for the dominant load frequency.

The computational domain is surrounded by AML on 3 sides with a width of $d_{i}=\lambda_{R}$, unless specified otherwise. The AML is terminated by Dirichlet boundary conditions. The material parameters are $E=60 \mathrm{MPa}, \nu=0.35$ and $\rho=1800 \mathrm{~kg} / \mathrm{m}^{3}$. The response of the pulse is captured in two observation points, $A^{+}$and $A^{-}$. The two observation points are located with equal distance to the load on the surface in the computational domain, very close to the AML interface.

The load is a Ricker pulse acting in a single point defined as

$$
P(t)=\tau\left(1-\tau^{2}\right)^{2}, \quad-1<\tau<1
$$


where $\tau=2 t / T-1$. The duration of the pulse is $0.2 \mathrm{~s}$, hence the dominant frequency of the pulse is $f=1 / T=5 \mathrm{~Hz}$. In the numerical examples, the maximum load is $P_{\max }=50 \mathrm{kN}$. The time history of the Ricker pulse and its Fourier content is given in Figure 4.
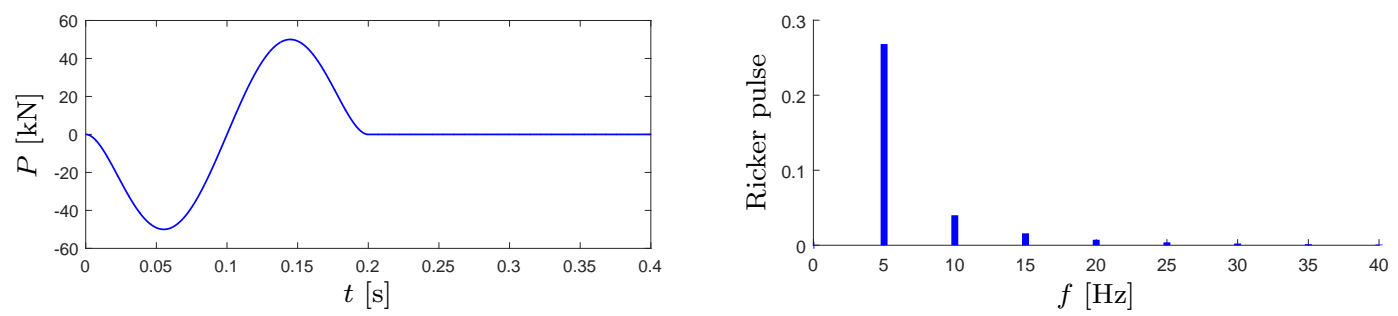

Figure 4: Ricker pulse and its Fourier spectrum.

In the time integration, the implicit version of the Newmark method is used, i.e. $\gamma=1 / 2$ and $\beta=1 / 4$ for unconditionally stable time marching. Although the time step is free of choice considering stability, it is essential to choose a time step that matches the temporal variation of the source. The choice of time step is therefore based on the CFL condition

$$
\Delta t=\min \left(\frac{1}{c_{p}}, \frac{1}{c_{s}}\right)\left(\frac{1}{\Delta x_{1}^{2}}+\frac{1}{\Delta x_{2}^{2}}\right)^{-1 / 2}
$$

as well as the previously mentioned condition $\Delta t \leq 1 /\left(8 f_{\max }\right)$, where $f_{\max }$ is the largest frequency to be represented in the time history.

The 2D FE-model is implemented in Matlab. However, the recursive update of the convolution terms is done by a Mex function, due to the faster computation of a for-loop running over each element in the AML region. The element length is found sufficient to be $2.57 \mathrm{~m}$ corresponding to 8 element s per Rayleigh wave length which is also suggested by $[22,23]$. In the two-layer half space where the top layer 
is thinner than the element width of $2.57 \mathrm{~m}$, this layer consist of thin, wide elements with thickness equal to the layer thickness. In all numerical examples a Rayleigh type damping is imposed with a structural damping ratio of $\zeta=0.01$ centered around the load frequency [24].

\subsection{Verification of $A M L$}

Three numerical examples are used to verify the AML in moving coordinates. The wave propagation in a half space subjected to the Ricker pulse moving along the surface (Figure 3) is calculated in the first example. The response is studied in observation points $A^{+}$and $A^{-}$for a half space truncated by AML, a half space with fixed boundaries and a reference value calculated in a large domain in which the waves do not reach the fixed boundaries within the simulation time. The traveling velocity of the pulse is $V \simeq 22 \mathrm{~m} / \mathrm{s}$. The calculated time period is 1 s and the time step is $0.0071 \mathrm{~s}$.

The time histories at the two observation points $A^{+}$and $A^{-}$are displayed in Figure 5. The responses computed using AML agree well with the responses computed in a large domain. The responses computed with fixed boundaries are seen to be off from the reference responses quite early. This proves the applicability of the AML even very close to the interface to AML.

The vertical displacement field is visualized with snapshots at four instants in time with AML and with fixed boundaries, respectively, in Figure 6. The traveling velocity of the pulse is $V \simeq 44 \mathrm{~m} / \mathrm{s}$. After $10 T_{c}$ corresponding to 2 seconds the wave has been fully absorbed in the AML (top row) while reflections of the wave are present at the same instants in time in case of fixed boundaries (bottom row).

The third example illustrates the response from a Ricker pulse traveling with velocity $V=22 \mathrm{~m} / \mathrm{s}$ in a moving frame of reference compared to the response from 
(a)

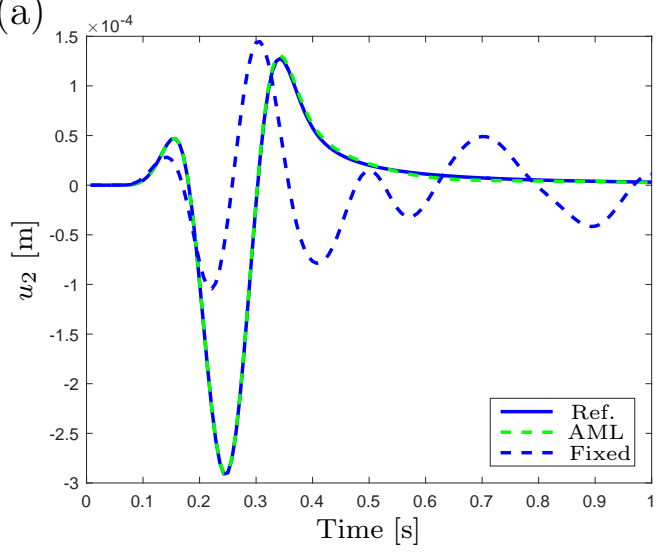

(b)

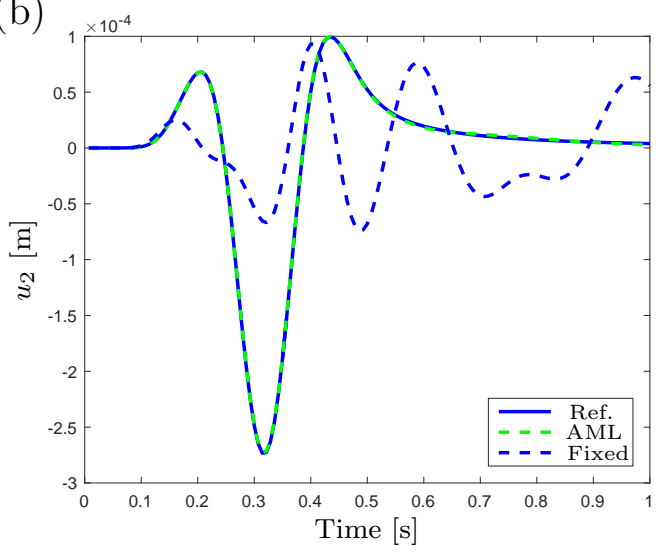

Figure 5: Vertical displacement at observation points, $A^{-}$and $A^{+}$.

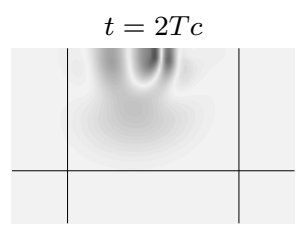

$t=2 T c$

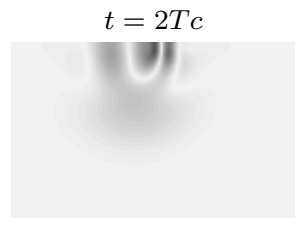

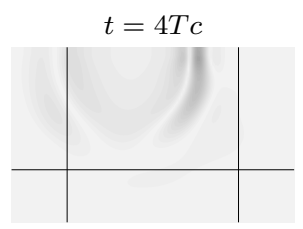

$t=4 T c$

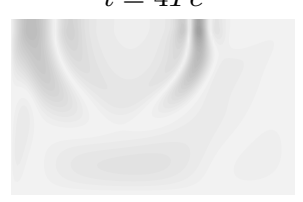

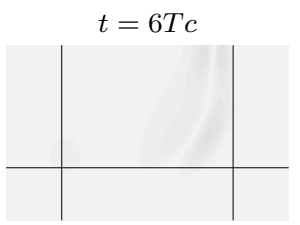

$t=6 T c$

$t=10 T c$

Figure 6: Snapshots of the vertical displacement field at four instances of time with Mach=0.4. Top row is with AML boundaries and bottom row is with fixed boundaries

manually moving the Ricker pulse in a static frame of reference (Figure 7 ). In this case the convection correction parameter is chosen to $h=0.4 l_{x}$ with $l_{x}$ being the side length of the element in the $x$-direction. The response is recorded at the distance $4 \mathrm{~m}$ 


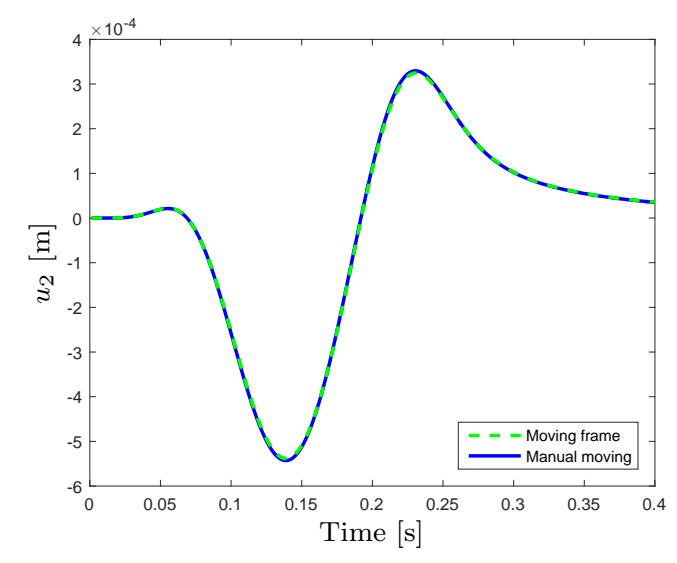

Figure 7: Response from Ricker pulse traveling with $V=22 \mathrm{~m} / \mathrm{s}$ in moving frame compared to static frame.

ahead of the load source. The response obtained in the moving frame of reference compares well with the response obtained in a static frame of reference.

\subsection{Parametric study of the AML parameters}

The absorbing properties of the AML are determined by a number of parameters. The choice of these parameters is essential for optimizing its performance. The spatial dependence of the AML attenuation function $\beta_{i}$ in the $x_{i}$ direction is chosen as a $2^{\text {nd }}$ order polynomial function as in $[8,20]$

$$
\beta_{i}=\beta_{i}^{\max }\left(\frac{x_{i}^{p}}{d_{i}}\right)^{2}, \quad i=1,2
$$

in which $x_{i}^{p}$ is measured from the interface to AML and $d_{i}$ is the thickness of the AML layer. The coefficient $\beta_{i}^{\max }$ is given by [8]

$$
\beta_{i}^{\max }=-\frac{3 c_{p} \log _{10}\left(R_{0}\right)}{2 d_{i}}
$$

where $R_{0}$ is the theoretical reflection coefficient at normal incidence and $c_{p}$ is the pressure wave velocity. Good performance of AML depends on proper selection of 
$\beta_{\max }$. Choosing it too small would result in pollution of the computational domain due to insufficient absorption. Choosing it too large will on the other hand result in spurious reflections from the interface due to inadequate representation of the AML by the discrete layer [20].

In this section, a parametric study of the free parameters in the attenuation function (49) is conducted to determine the optimal parameters for obtaining good accuracy and efficient computations.

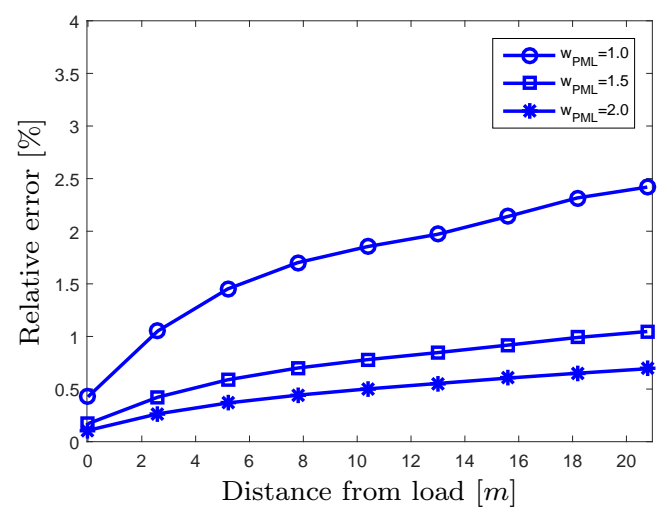

Figure 8: Maximum relative error at a number of observation points computed using the AML widths $\mathrm{w}_{\mathrm{PML}}=d_{i} / \lambda_{c_{r}}=1.0,1.5,2.0$.

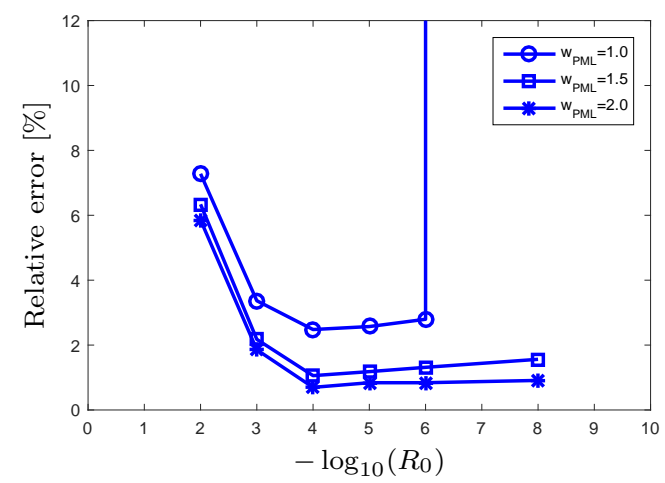

Figure 9: Maximum relative error as function of $R_{0}$ for each AML width. $n=2$. 
The attenuation function has 3 parameters; $c_{p}, R_{0}$ and $d_{i}$ where for simplicity $d_{1}=d_{2}$. The pressure wave velocity $c_{p}$ is given by the material properties of the medium, leaving 2 parameters to be determined. A parameter study on these 2 parameters has been conducted, and the results are shown in Figure 8-9. The results are expressed as the maximum relative error between the transient response from the Ricker pulse using AML and the reference value introduced in the previous section, calculated as

$$
\text { relative error }=\frac{\max \left|U^{P M L}-U^{r e f}\right|}{\max \left(U^{r e f}\right)}
$$

where $U$ is the transient response obtained at a certain observation point, and the exponents denotes whether the response is obtained using AML or it is the reference value. The simulations have been running for $1.5 \mathrm{~s}$ ensuring enough time for the waves to reflect back to the origin of the load source for the thickest AML layer of interest. All tests are done for the AML thicknesses $d_{i}=0.5 \lambda_{c_{r}}, \lambda_{c_{r}}, 1.5 \lambda_{c_{r}}$ and $2 \lambda_{c_{r}}$. However, a AML width of $0.5 \lambda_{c_{r}}$ turns out to be too thin and the response diverges. The errors obtained from using this thin layer are therefore not presented in Figures 8 and 9.

Figure 8 illustrates the performance of the AML with the different AML layer thicknesses. The response is obtained at a number of observation points on the surface from under the load source to the interface of AML. The spacing between the observation points is one element length. The reflection coefficient is $R_{0}=10^{-4}$. For a AML width of $d_{i}=\lambda_{c_{r}}$, the relative error under the load source is $<0.5 \%$ increasing to $2.5 \%$ at the interface to AML. The relative errors for $d_{i}=1.5 \lambda_{c_{r}}$ and $d_{i}=2 \lambda_{c_{r}}$ varies closely between $0-1 \%$ from under the load source to the AML interface. This corresponds to an absolute error of 4-5 $\mu \mathrm{m}$ for $d_{i}=\lambda_{c_{r}}$ and 1-2 $\mu \mathrm{m}$ for $d_{i}=1.5 \lambda_{c_{r}}$. Whether to choose $d_{i}=\lambda_{c_{r}}$ or $d_{i}=1.5 \lambda_{c_{r}}$ comes down to a balance 
between accuracy and computational efficiency. In the further examples of this paper, $d_{i}=1.5 \lambda_{c_{r}}$ is used.

In Figure 9, the relative error from the variation of the reflection coefficient between $R_{0}=10^{-2}-10^{-8}$ is illustrated for the different widths. This figure shows for all thicknesses that the best choice of reflection coefficient lies in the area of $R_{0}=10^{-4}$. Choosing it any smaller will not improve the result and will at some point lead to a divergence of the response (as seen for $d_{i}=\lambda_{c_{r}}$ with $R_{0}<10^{-6}$. Choosing a larger $R_{0}$ results in a significant increase of the maximum relative error. In the further numerical examples, $R_{0}=10^{-4}$ is used.

The following examples in this paper are based on the reflection coefficient $R_{0}=$ $10^{-4}$ and the AML width $d_{i}=1.5 \lambda_{c_{r}}$ corresponding to 8 elements.

\subsection{Single layer half space}

The simplest case for testing the performance of the AML is to apply a Ricker pulse load to a single layer half space (Figure 3). The pulse is traveling on the surface with different velocities in the horizontal direction. The velocity is expressed in relation to the shear wave velocity of the soil as the Mach value

$$
\text { Mach }=V / c_{s}
$$

The response is obtained in Figure 10 at Mach $0,0.2$ and 0.4 . The pulse arrives with the speed $c+V$ behind the load, point $A^{-}$, and it arrives with the speed $c-V$ in front of the load, at point $A^{+}$. I.e. for a fixed point in front of the moving load the frequency increases while it decreases for a fixed point behind the moving load.

Hence, the wave length defined by $c / f$ is changing according to the change in speed, given by

$$
f=\left(\frac{c}{c+V}\right) f_{0}
$$


(a)

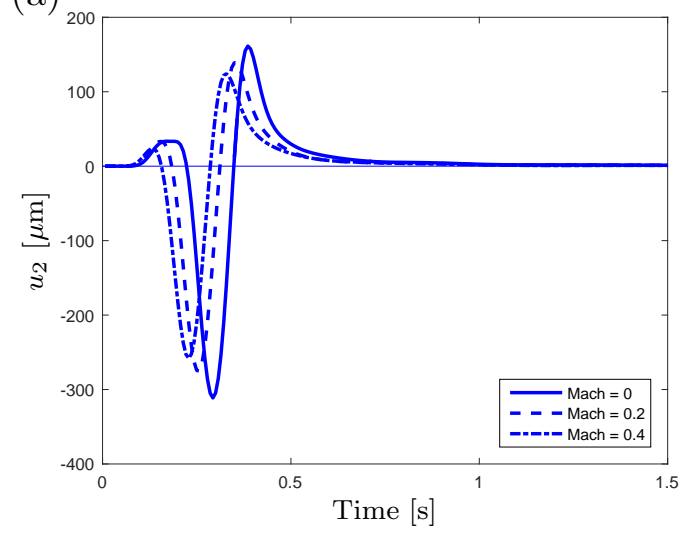

(b)

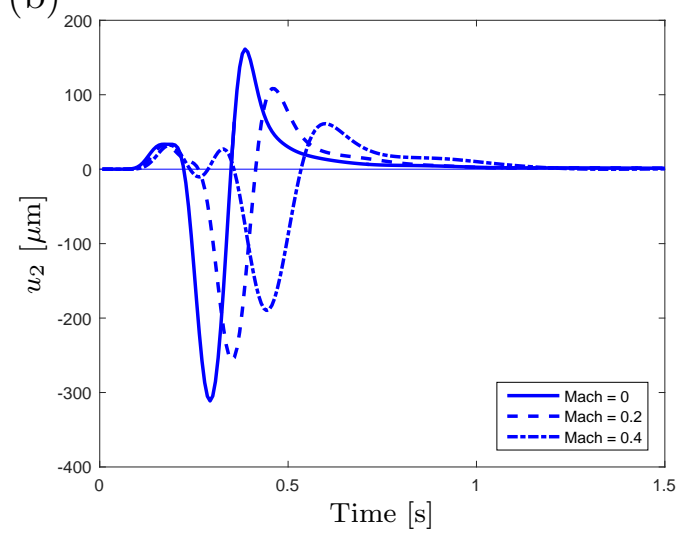

Figure 10: Deflection response in points $A^{-}$(a) and $A^{+}$(b) for single layer system.

where $c$ is the wave velocity of the medium, $V$ is velocity of the pulse and $f_{0}$ is the frequency of the load. It follows from this equation that by increasing the speed of the source, the resulting frequency is spread over a wider range of frequencies. Hence, the AML width needs to be chosen such that the shortest wavelength is ensured a resolution of 8 elements.

The dynamic response is seen to decrease with velocity (Fig. 10). This is especially clear in front of the load where the maximum deflection decreases by approximately $30 \%$. This is in agreement with the results found by [16]. An opposite observation can be made in case of a constant moving load. In this case, the response increases by increasing velocity $[25,26]$.

The Ricker pulse traveling with velocities Mach 0, 0.2 and 0.4 is fully absorbed by the AML layer in the single layer half space. 


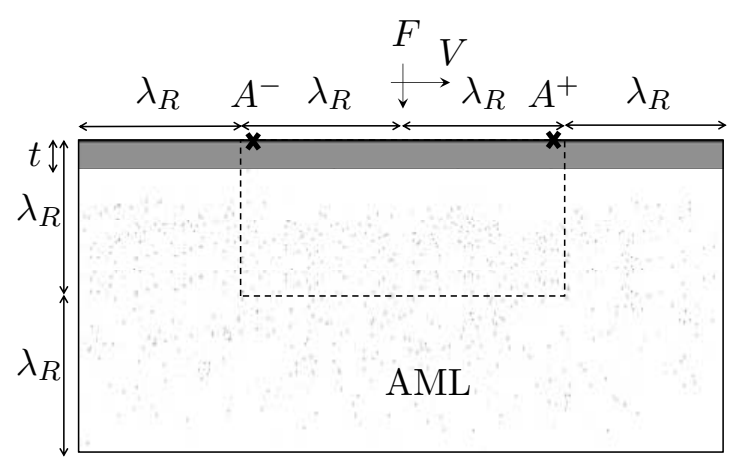

Figure 11: Domain of interest with stiff top layer truncated by AML.

\subsection{Two layer half space}

In this example the effect of two layers on the response of a Ricker pulse is studied. The pulse is the one illustrated in Figure 4 used in the previous example. A $100 \mathrm{~mm}$ stiff layer is added on top of the single layer treated in section 5.3 as sketched in Figure 11. The density of the top layer is $\rho=2300 \mathrm{~kg} / \mathrm{m}^{3}$ and Poisson's ratio is $\nu=0.35$. The effect of impedance ratio is analysed in the following examples where Young's modulus of the top layer is chosen to $E, 10 E$ and $100 E$, respectively, where $E=60 \mathrm{MPa}$ is the modulus of the bottom layer. The response in point $A^{+}$and $A^{-}$obtained from the Ricker load of zero velocity acting on the two layer system with varying top layer stiffness is seen in Figure 12. The first two peaks of the response are seen to increase with increasing impedance ratio. Also, the wave period becomes shorter as the impedance ratio increases. This is explained from the fact that the wave period is given by $T=\lambda / c$. The wave velocity of the two-layer system is a combination of the wave velocities of the top and the bottom layer. Since the wave velocity in the top layer is larger than that of the bottom layer, the wave velocity of the system will increase with increasing impedance ratio. Hence, 


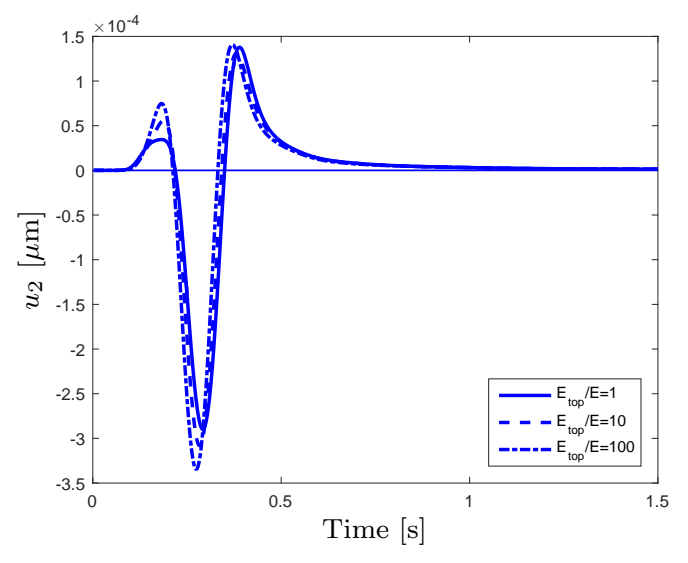

Figure 12: Response from Ricker wavelet acting on a two layer system with top layer stiffness $E$, $10 E$ and $100 E$ at $\mathrm{Mach}=0$.

the wave period will decrease. The response in point $A^{+}$equals the response in point $A^{-}$due to symmetric wave propagation.

(a)

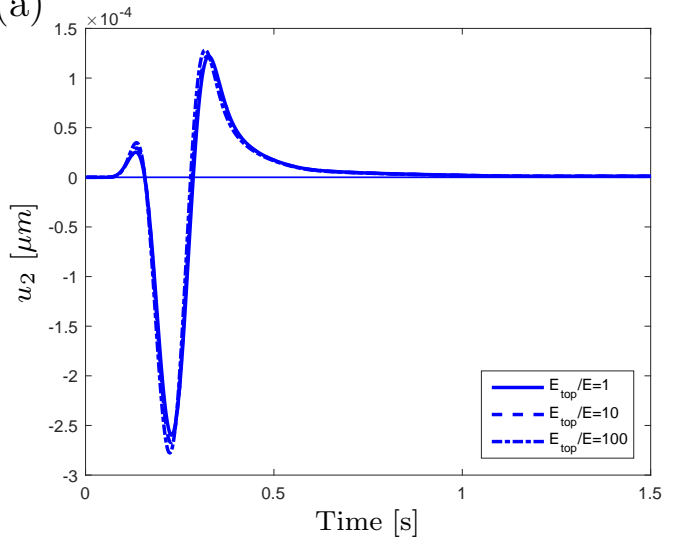

(b)

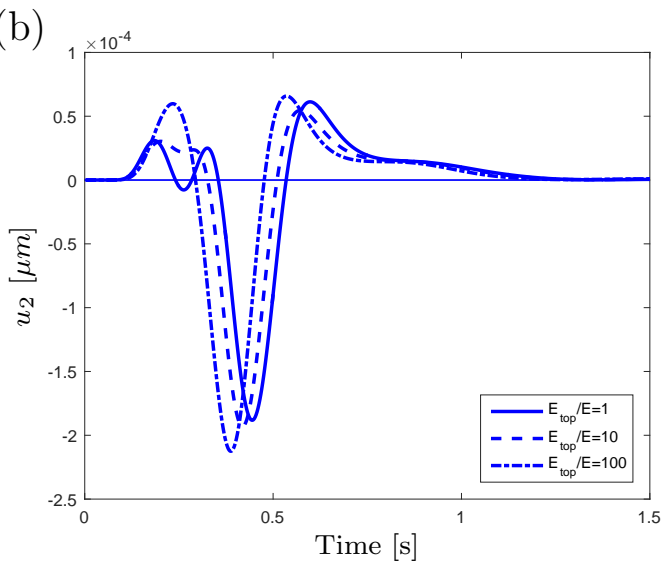

Figure 13: Deflection response in points $A^{-}$(a) and $A^{+}$(b) for a two layer system with top layer stiffness $E, 10 E$ and $100 E$ at $\mathrm{Mach}=0.4$.

Increasing the velocity of the load to Mach $=0.4$ yields the responses in point $A^{+}$and $A^{-}$given in Figure 13. A high impedance ratio is seen to have a significant 
influence on the response obtained in front of the load (Figure 13b). The negative displacement peak increases around $13 \%$ when the impedance ratio equals 100 . This peak also occurs approximately $0.05 \mathrm{~s}$ earlier compared to the response obtained in the single layer example. On the other hand, the response obtained behind the load does not change substantially. The effects observed for the response in front of the load are only seen to minor degree behind the load.

In a two-layer system $c_{p}$ in the subgrade is still dominating the wave velocity, hence this value is valid for use in the attenuation function in the AML layer.

\section{Conclusions}

An approximate form of the Perfectly Matched Layer (PML), here termed the Asymptotically Matched Layer, has been formulated in a moving frame of reference. With this formulation, the FE-model can be limited to the domain of interest yielding computational efficiency.

The AML demonstrates great absorbing abilities and numerical examples verifies that an accurate response can be obtained very close to the AML interface. The parametric study of the AML parameters showed that a AML width of 1.5 Rayleigh wave length with respect to the dominating load frequency is sufficient to achieve good accuracy in the response, i.e. a maximum relative error of less than $1 \%$ close to the interface to the AML layer. The optimal reflection coefficient to solve the treated problem was found to be around $R_{0}=10^{-4}$.

Numerical examples have been conducted for a single- and a two-layer half space. These examples clarifies that the wave propagation is dominated by the properties of the subgrade in the pavement. Hence the AML parameters can in a multi layered pavement system be set according to the properties of the subgrade. 
The stiffness ratio between top layer and the underlying layer has a significant effect on the response obtained in front of the load. The response is seen to increase with increasing stiffness of the top layer and the arrival time of the response decreases.

\section{Acknowledgements}

This work is supported by the Innovation Fund Denmark through the grant 'High-Speed Pavement Assessment Tool for Better and Safer Roads'.

\section{References}

[1] J.-P. Berenger, A perfectly matched layer for the absorption of electromagnetic waves, Journal of Computational Physics 114 (1994) 185-200.

[2] W. C. Chew, W. H. Weedon, A 3D perfectly matched medium from modified Maxwell's equations with stretched coordinates, Microwave and Optical Technology Letters 7 (1994) 599-604.

[3] W. C. Chew, Q. H. Liu, Perfectly matched layers for elastodynamics: A new absorbing boundary condition, Journal of Computational Acoustics 4 (1996) $341-359$.

[4] U. Basu, A. K. Chopra, Perfectly matched layers for time-harmonic elastodynamics of unbounded domains: Theory and finite-element implementation, Computer Methods in Applied Mechanics and Engineering 192 (2003) 13371375. 
[5] U. Basu, A. K. Chopra, Perfectly matched layers for transient elastodynamics of unbounded domains, International Journal for Numerical Methods in Engineering 59 (2004) 1039-1074.

[6] U. Basu, Explicit finite element perfectly matched layer for transient threedimensional elastic waves, International Journal for Numerical Methods in Engineering 77 (2) (2009) 151-176.

[7] S. Kucukcoban, L. F. Kallivokas, Mixed perfectly-matched-layers for direct transient analysis in 2D elastic heterogeneous media, Computer Methods in Applied Mechanics and Engineering 200 (2011) 57-76.

[8] R. Matzen, An efficient finite element time-domain formulation for the elastic second-order wave equation: A non-split complex frequency shifted convolutional PML, International Journal for Numerical Methods in Engineering 88 (2011) 951-973.

[9] E. Bécache, S. Fauqueux, J. P, Stability of perfectly matched layers, group velocities and anisotropic waves, Journal of Computational Physics 188 (2003) 399-433.

[10] K. Duru, G. Kreiss, Numerical interaction of boundary waves with perfectly matched layers in two space dimensional elastic wave guides, Wave Motion 51 (2014) 445-465.

[11] K. Duru, G. Kreiss, Boundary waves and stability of the perfectly matched layer for the two space dimensional elastic wave equation in second order format, SIAM Journal of Numerical Analysis 52 (2014) 2883-2904. 
[12] P. Yoo, I. Al-Qadi, Effect of transient dynamic loading on flexible pavements, Transportation Research Record: Journal of the Transportation Research Board 1990 (2007) 129-140.

[13] H. Wang, I. Al-Qadi, S. Portas, M. Coni, Three-dimensional finite element modeling of instrumented airport runway pavement responses, Transportation Research Record: Journal of the Transportation Research Board 2367 (2013) 76-83.

[14] H. Lane, P. Kettil, N.-E. Wiberg, Moving finite elements and dynamic vehicle interaction, European Journal of Mechanics - A/Solids 27 (4) (2008) 515-531.

[15] H. A. Dieterman, V. Metrikine, Steady-state displacements of a beam on an elastic half-space due to a uniformly moving constant load, European Journal of Mechanics - Series A/Solids 16 (1997) 295-306.

[16] S. Krenk, L. Kellezi, S. R. K. Nielsen, P. Kirkegaard, Finite element and transmitting boundary conditions for moving loads, in: Proceedings of the 4th European Conference on Structural Dynamics, Eurodyn'99, Balkema, The Netherlands, 1999, pp. 447-452.

[17] L. Andersen, S. R. K. Nielsen, S. Krenk, Numerical methods for analysis of structure and ground vibration from moving loads, Computers \& Structures 85 (2007) 43-58.

[18] J. Diaz, J. P, A time domain analysis of PML modfels in acoustics, Computer Methods in Applied Mechanics and Engineering 195 (2006) 3820-3853.

[19] Y. Zheng, X. Huang, Anisotropic perfectly matched layers for elastic waves in 
Cartesian and curvilinear coordinates, Tech. rep., Massachusetts Institute of Technology, Earth Resources Laboratory (2002).

[20] I. Harari, U. Albocher, Studies of FE/PML for exterior problems of timeharmonic elastic waves, Computer Methods in Applied Mechanics and Engineering 195 (2006) 3854-3879.

[21] N. M. Newmark, A method of computation for structural dynamics., Journal of the Engineering Mechanics Division, ASCE 85.

[22] W. Zhai, E. Song, Three dimensional FEM of moving coordinates for the analysis of transient vibrations due to moving loads, Computers and Geotechnics 37 (2010) 164-174.

[23] J. Lysmer, Analytical procedures in soil dynamics, Tech. Rep. UCB/EERC78/29, Earthquake Engineering Research Center, University of California, Berkeley.

[24] P. Vinh, R. Ogden, On formulas for the Rayleigh wave speed, Wave Motion 39 (2004) 191-197.

[25] A. V. Metrikine, H. A. Dieterman, Lateral vibrations of an axially compressed beam on an elastic half-space due to a moving lateral load, European Journal of Mechanics - A/Solids 18 (1999) 147-158.

[26] H. H. Hung, Y. B. Yang, Elastic waves in visco-elastic half-space generated by various vehicle loads, Soil Dynamics and Earthquake Engineering 21 (2001) $1-17$. 